\title{
Influence of single vacancy defect at varying length on electronic properties of zigzag graphene nanoribbons
}

\author{
Kien Liong Wong, Mu Wen Chuan, Wee Khang Chong, Afiq Hamzah, Mohd Shahrizal Bin Rusli, \\ Nurul Ezaila Binti Alias, Cheng Siong Lim, Michael Loong Peng Tan \\ School of Electrical Engineering, Faculty of Engineering, Universiti Teknologi Malaysia, Malaysia
}

\begin{tabular}{l} 
Article Info \\
\hline Article historys: \\
Received Mar 25, 2019 \\
Revised Apr 28, 2019 \\
Accepted May 10, 2019 \\
\hline
\end{tabular}

\section{Keywords:}

Graphene nanoribbons

Hamiltonian

Non-equilibrium green's

function

Tight binding

Vacancy defect

\begin{abstract}
Graphene, identified in 2004, is now an established two-dimensional (2D) material due to its outstanding physical and electronic characteristics namely its superior electrical conductivity. Graphene is a zero-gap material that has linear dispersion with electron-hole symmetry. As pristine sheet, it cannot be utilized in digital logic application without the induction of a band gap inside the band structure. In our work, the modeling and simulation of graphene nanoribbons (GNRs) are carried out to determine its electronics properties that are benchmarked with other published simulation data. A 4-Zigzag GNRs (4-ZGNRs) under different length are utilized. A single vacancy defects is introduced at various positions inside the atomic structure. The theoretical model is implemented based on single-neighbour tight binding technique coupled with a non-equilibrium Green's function formalism. The single vacancy defects are represented by the elimination of tight binding energies in the Hamiltonian matrix. Subsequently, these matrix elements are utilized to compute dispersion relation and density of states (DOS) through Green's function. It is found that single vacancy defects at different positions in 4-ZGNRs' atomic structure under varying length has no significant impacts on the sub-band structure but these vacancies impact the DOS that are computed throught Green's function approach.
\end{abstract}

Copyright $(2019$ Institute of Advanced Engineering and Science. All rights reserved.

\section{Corresponding Author:}

Michael Loong Peng Tan,

School of Electrical Engineering, Faculty of Engineering,

Universiti Teknologi Malaysia,

81310 Skudai, Johor, Malaysia.

Email:michael@utm.my

\section{INTRODUCTION}

Graphene Nanoribbons (GNRs) or strips of graphene are theoretically introduced by Mitsutaka Fujita in 1997 [1]. GNRs are one of the potential material that can complement and ultimately replace Silicon [2] because of its remarkable electronic properties that can be engineered by controlling its width, length and edge orientation as well as defects [3,4]. Defects can be a beneficial advantage that can alter the electronics properties to enhance their applicability for a distinctive applications [5]. In tight binding approach, Hamiltonian matrix that is a solution of Schrödinger's equation is obtained to compute electronic properties of 4-ZGNRs with single vacancy defects at different locations inside the band structure [6-8]. The nanoribbon system can be described by Hamiltonian matrix the elements [9, 10]. A tight binding 4-ZGNR model is produced by disintegrating 4-ZGNRs' quasi-2D structure into an equivalent one-dimensional (1D) matrix system with alpha and beta matrices [11,12]. Figure 1 depicts an imperfect 4-ZGNRs at length of 3 unit cells with single vacancy defect at intersection of first row and third column. 


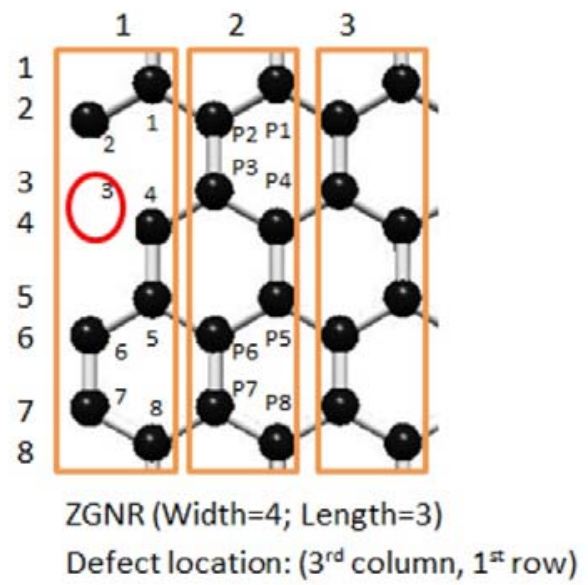

Figure 1. (a) Atomic structure of 4-ZGNRs at length of 3 unit cells for defect location at first row of third column intersection

\section{RESEARCH METHOD}

Non-ideal ZGNRs with single vacancy defect is implemented through single neighbor tight-binding approach within non-equilibrium Green's function formalism in MATLAB. Tight-binding method uses semiempirical model for simple and fast computation. The construction of alpha matrix, $\alpha$ that consists of the interaction among atoms within the unit cell, is shown in (1). The (2) shows Beta matrix, $\beta$ that encapsulates the interaction between unit cell $[13,14]$. Parameter $t$ is the tight binding energy between carbon atoms and $2.7 \mathrm{eV}$ are used for nearest neighbout [15]. The red zeroes in Equation 1 highlights the affected tight-binding energies that are eliminated when a carbon atom is vacated. The (3) shows the Hamiltonian operator with the inclusion of $\alpha$ and $\beta$ matrices. The $\alpha$ matrix of each unit cell is located at the diagonal of Hamiltonian matrix whereas the $\beta$ matrix is located at the top of the diagonal matrix. Parameter $\beta$ ' matrix is the transpose of $\beta$ matrix located at the bottom of diagonal.

$$
\alpha_{4-Z G N R}=\begin{array}{cccccccc}
1 & 2 & 3 & 4 & 5 & 6 & 7 & 8 \\
2 & 3 \\
& 4 \\
& 6 \\
7 & 8 \\
8
\end{array}\left[\begin{array}{llllllll}
0 & t & 0 & 0 & 0 & 0 & 0 & 0 \\
t & 0 & 0 & 0 & 0 & 0 & 0 & 0 \\
0 & 0 & 0 & 0 & 0 & 0 & 0 & 0 \\
0 & 0 & 0 & 0 & t & 0 & 0 & 0 \\
0 & 0 & 0 & t & 0 & t & 0 & 0 \\
0 & 0 & 0 & 0 & t & 0 & t & 0 \\
0 & 0 & 0 & 0 & 0 & t & 0 & t \\
0 & 0 & 0 & 0 & 0 & 0 & t & 0
\end{array}\right]
$$

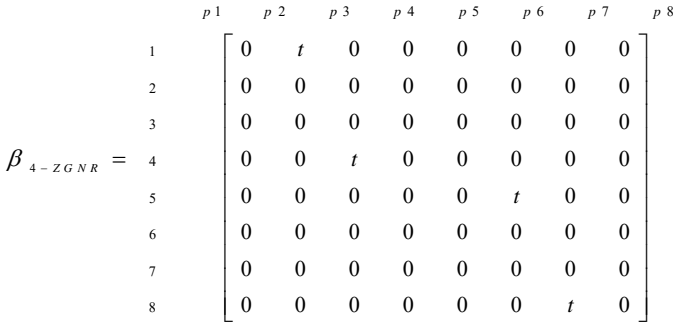

$$
H_{4-A G N R / Z G N R, L=3}=\left[\begin{array}{ccc}
\alpha_{11} & \beta_{12} & 0 \\
\beta^{\prime}{ }_{21} & \alpha_{22} & \beta_{23} \\
0 & \beta_{32}^{\prime} & \alpha_{33}
\end{array}\right]
$$


The periodic boundary condition is the applied to obtain the the Hamiltonian operator matrix in (3). Following that, the energy and wave number relationship is given by the dispersion relation in (4) where $\alpha$ and $\beta$ matrices of (1) and (2) are used respectively to yield the system energy sub-band structure $[13,16]$.

$$
E=\alpha+\beta^{\prime} e^{-i k a}+\beta e^{i k a}
$$

The availability of energy states can be shown through the density of states (DOS) in (5) [17] that required non-equilibrium Green's function (NEGF) formalism

$$
\begin{aligned}
& \operatorname{DOS}(E)=\frac{1}{\pi} \operatorname{Im}\left[\operatorname{Trace}\left[G_{F}\right]\right] \\
& G_{F}=[(E+i \eta) * I-H]^{-1}
\end{aligned}
$$

where $G_{F}$ is retarded Green's function, $\eta$ is a very small imaginary value to block divergence of the inverse matrix, $I$ is an identity matrix, and $H$ is the Hamiltonian operator matrix [18].

\section{RESULTS AND DISCUSSION}

First, the band structure of pristine ZGNRs is simulated and compared with previously reported data on the band structure. Figure 2 illustrates the band structure of 4-ZGNRs and 5-ZGNRs with previously reported studies that also adopted tight binding approach $[19,20]$. It is demonstrated that our results are in good agreement with the previously published data. Figure 3 shows the band structure of 8-ZGNRs generated using tight-binding method versus Density Functional Theory (DFT) method [21]. As expected, there are differences in band structure as our 8-ZGNR does not have any bandgap whereas DFT shows a small bandgap. DFT method are used to determine the ground state properties of an electronic system from the electron density and suitable for a large and complex systems. Nevertheless, tight binding is sufficient for a simple system and less computationally expensive than DFT approaches.

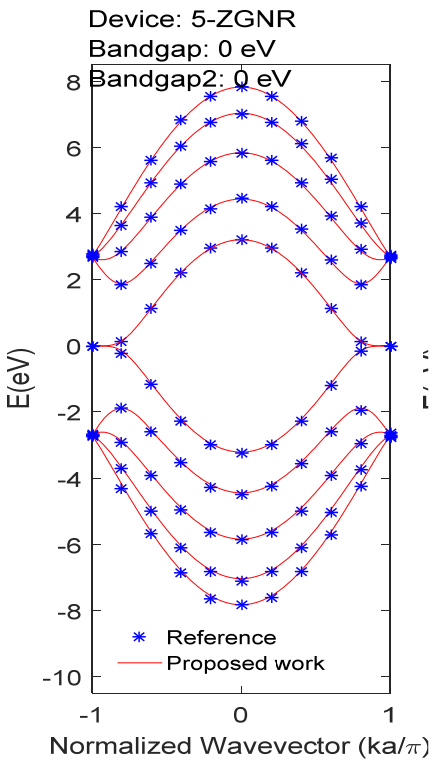

(a) 5-ZGNRs

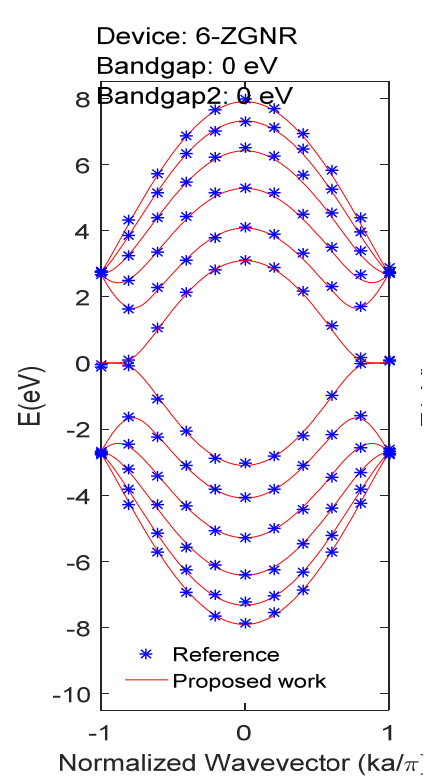

(b) 6-ZGNRs

Figure 2. Band structure of pristine (a) 5-ZGNRs; (b) 6-ZGNRs using tight binding method versus previously reported data $[19,20]$

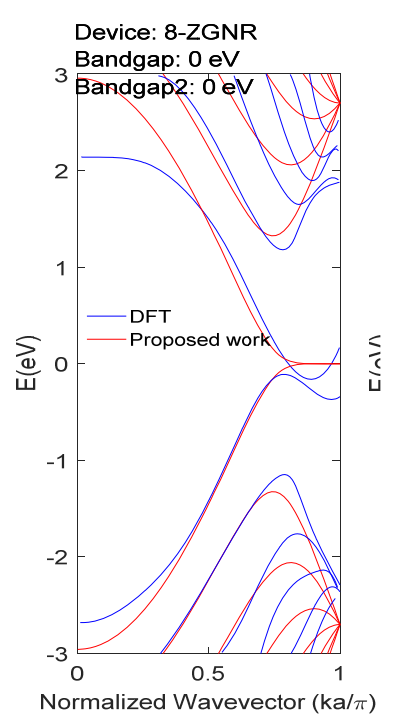

Figure 3. Band structure of pristine 8-ZGNRs using tight binding method versus DFT method [21] 
Next, vacancy defect is then introduced in the atomic structure of ZGNRs and the electronics properties of non-ideal nanoribbon is explored. The position of the single vacancy defect inside atomic structure is defined in terms of a row versus column plane. Figure 4 (a) and Figure 5 (a) depict a non-ideal 4-ZGNRs at length of 3 unit cells with single vacancy defect at different locations. A pristine 4-ZGNR with length of 3 unit cells has 24 carbon atoms inside the atomic structure. The single vacancy defect decreases the number of carbon atoms to 23. In Figure 4 (a), a single carbon atom is absent at the first row of third column intersection along the edge of 4-ZGNR atomic structure. In Figure 5 (a), a single carbon atom is missing at the second row of third column intersection within the bulk of 4-ZGNR atomic structure. Red circle in the figure indicates the absence of atoms inside the atomic structure. The interaction to previously neighbouring carbon atoms no longer exist thus the tight bonding energies are completely removed from the 4-ZGNR Hamiltonian matrix elements. Finally, dispersion relation and Green's function are computed to study the non-ideal 4-ZGNRs' electronic properties at varying defect locations particularly the sub-band structure in Figure 4 (b) and Figure 5 (b) and Green's function DOS in Figure 4 (c) and Figure 5 (c) respectively.

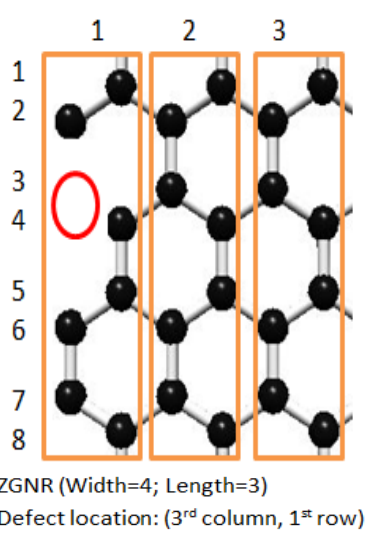

(a) Atomic structure

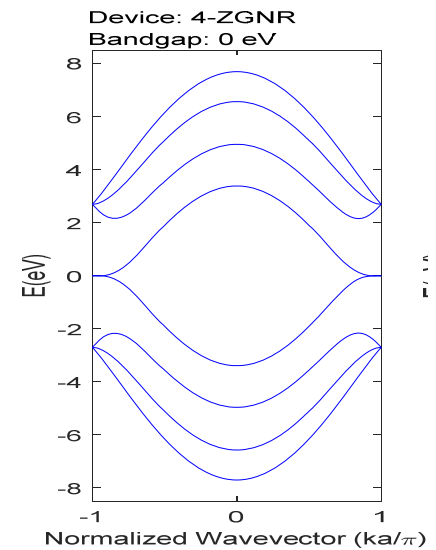

(b) Sub-band plot

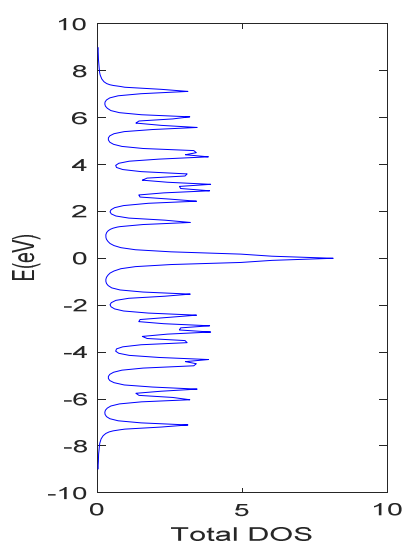

(c) Green's function DOS plot

Figure 4. (a) Atomic structure of 4-ZGNRs at length of 3 unit cells for defect location at first row of third column intersection; (b) Sub-band plot; (c) Green's function DOS plot of 4-ZGNRs as in (a)

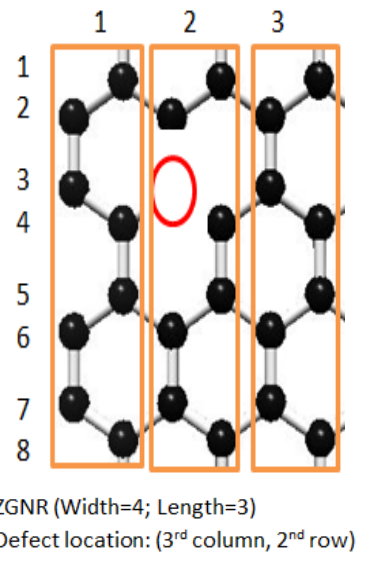

(a) Atomic structure

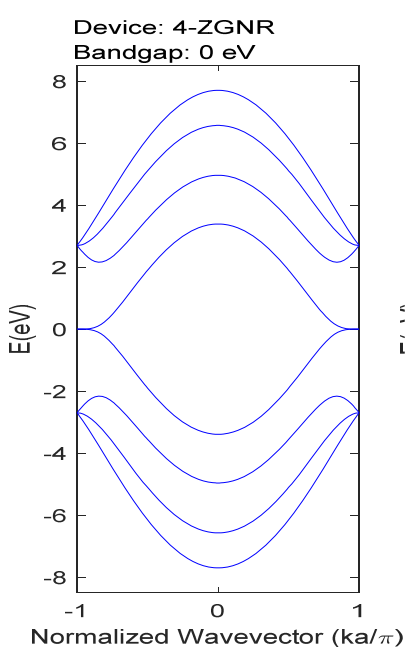

(b) Sub-band plot

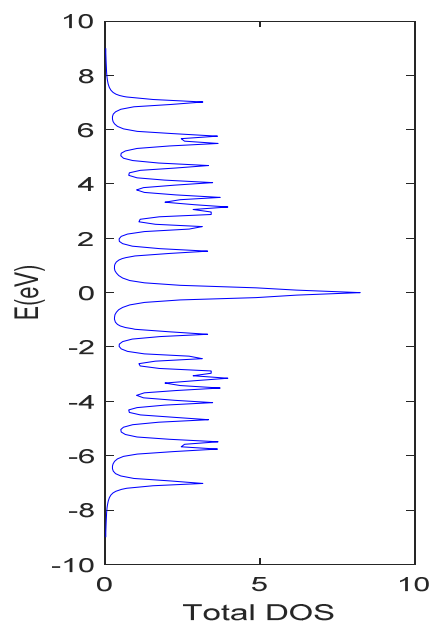

(c) Green's function DOS plot

Figure 5. (a) Atomic structure of 4-ZGNRs at length of 3 unit cells for defect location at second row of third column intersection; (b) Sub-band plot; (c) Green's function DOS plot of 4-ZGNRs as in (a) 
A zero band gap is observed of non-ideal 4-ZGNR at length of 3 unit cells and thus behaves like a metal. Figure 4 (b) and Figure 5 (b) reveals that the sub-band structure is unaffected by varying positions of single vacancy defect in 4-ZGNRs' atomic structure. The peak values for the sub-band are consistent across all configurations which are around $\pm 8 \mathrm{eV}$. However, vacancies directly affect the Green's function DOS of Figure 4 (c) and Figure 5 (c) where each DOS has distinct number of peaks and shapes. For instance, the peak numbers for DOS inside Figure 4 (c) and Figure 5 (c) are 19 and 20 respectively. In addition, whenever there is a vacancy defect along the edges or inside the bulk of 4-ZGNRs, there is a peak arising at $0 \mathrm{eV}$. A non-ideal 4-ZGNRs at length of 4 unit cells with single vacancy defect at different locations inside atomic structure are shown in Figure 6 (a) and Figure 7 (a). A pristine 4-ZGNR with length of 4 unit cells has 32 carbon atoms inside the atomic structure but reduces to 31 when vacancy is introduced. A carbon atom is missing at the intersection of first row of third column in Figure 6 (a) and at the intersection of second row and third column in Figure 7 (a). The absence of atom is represented by the red circle. Dispersion relation and Green's function DOS of a non-ideal 4-ZGNRs are shown in Figure 6 (b) and Figure 7 (b) as well as Figure 6 (c) and Figure 7 (c) respectively.

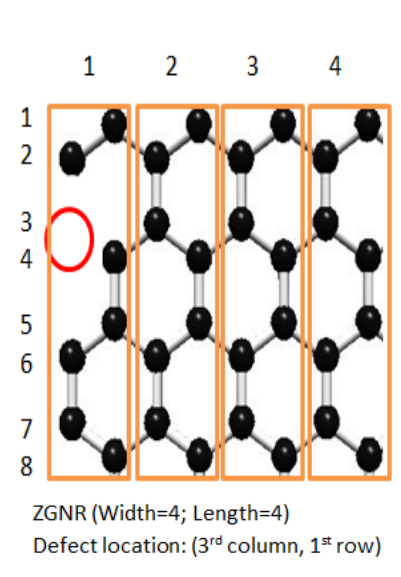

(a) Atomic structure

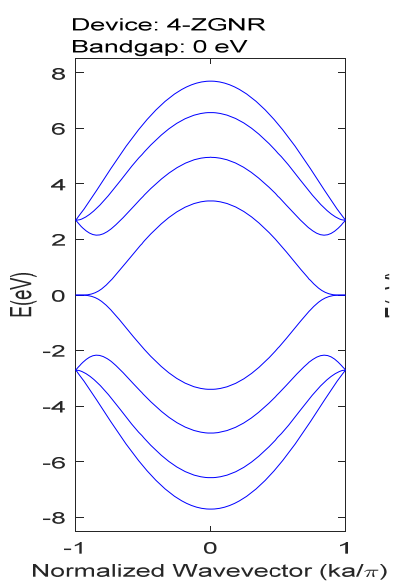

(b) Sub-band plot

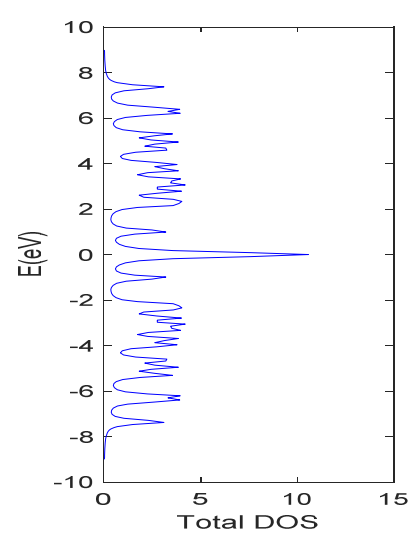

(c) Green's function DOS plot

Figure 6. (a) Atomic structure of 4-ZGNRs at length of 4 unit cells for defect location at first row of third column intersection; (b) Sub-band plot; (c) Green's function DOS plot of 4-ZGNRs as in (a)

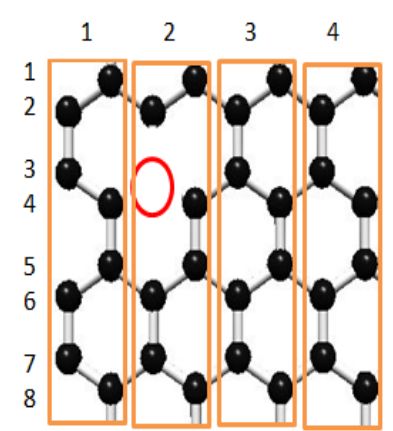

ZGNR (Width=4; Length=4)

Defect location: ( $3^{\text {rd }}$ column, $2^{\text {nd }}$ row)

(a) Atomic structure

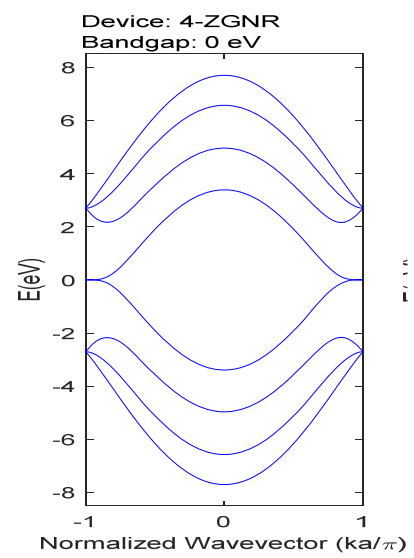

(b) Sub-band plot

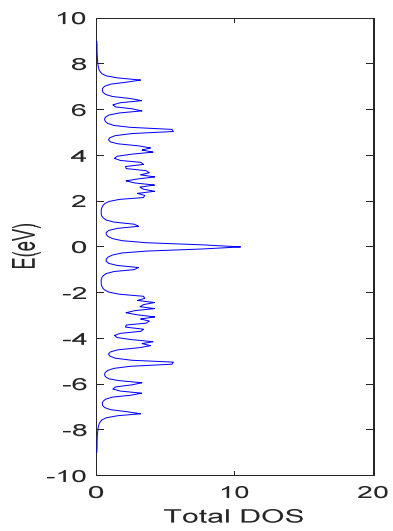

(c) Green's function DOS plot

Figure 7. (a) Atomic structure of 4-ZGNRs at length of 4 unit cells for defect location at second row of third column intersection; (b) Sub-band plot; (c) Green's function DOS plot of 4-ZGNRs as in (a) 
The DOS of Figure 7 (c) has 4 extra number of peaks compared with DOS inside Figure 6 (c). Moreover, when vacancy defect is located at the edge of 4-ZGNR, the peak value of highest DOS peak of Figure 6 (c) is lower by $5 \%$ than the highest peak of Figure 7 (c) where the single vacancy defect is located in the bulk. The non-ideal 4-ZGNRs at length of 5 unit cells with single vacancy defect at different locations inside atomic structure are illustrated in Figure 8 (a) and Figure 9 (a) where there are 39 carbon atom. A carbon atom is missing at the intersection of first row of third column in Figure 8 (a) and at the intersection of third row of third column in Figure 9 (a). Dispersion relation and Green's function DOS of a non-ideal 4-ZGNRs are shown in Figure 8 (b) and Figure 9 (b) as well as Figure 8 (c) and Figure 9 (c) respectively.

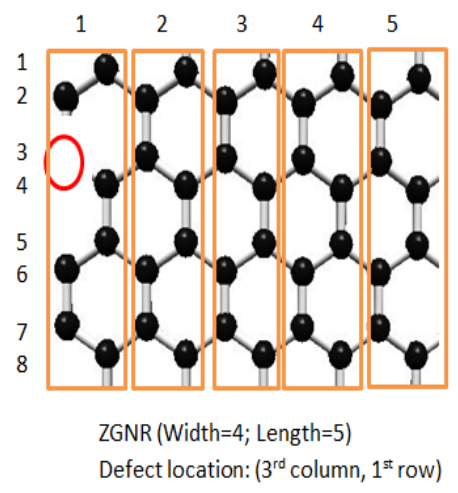

(a) Atomic structure

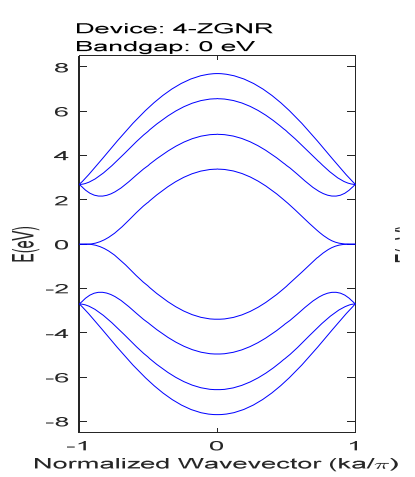

(b) Sub-band plot

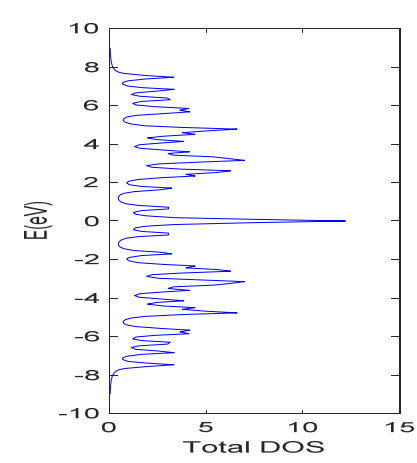

(c) Green's function DOS plot

Figure 8. (a) Atomic structure of 4-ZGNRs at length of 5 unit cells for defect location at first row of third column intersection; (b) Sub-band plot; (c) Green's function DOS plot of 4-ZGNRs as in (a)

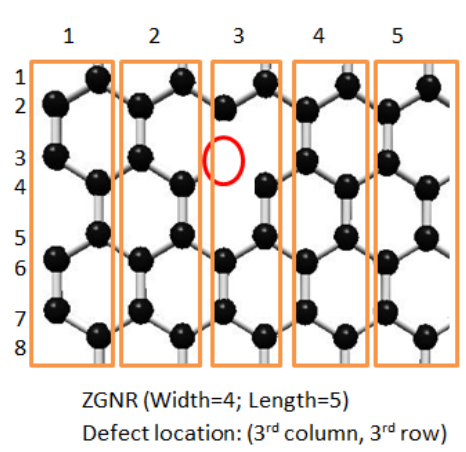

(a) Atomic structure

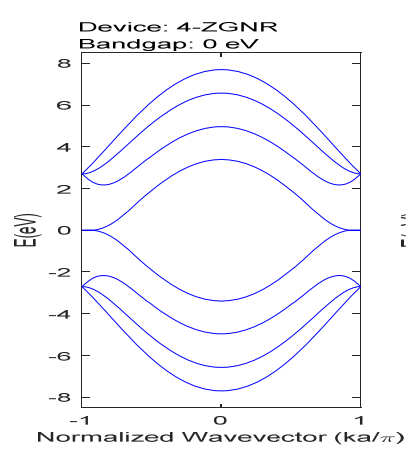

(b) Sub-band plot

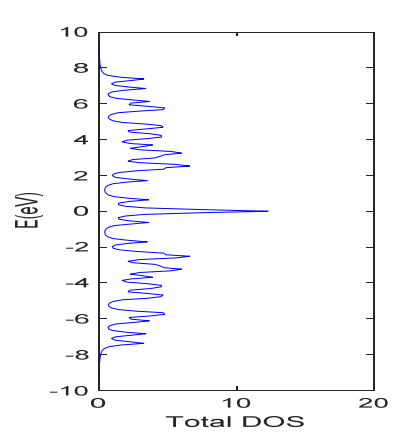

(c) Green's function DOS plot

Figure 9. (a) Atomic structure of 4-ZGNRs at length of 5 unit cells for defect location at third row of third column intersection; (b) Sub-band plot; (c) Green's function DOS plot of 4-ZGNRs as in (a)

For 4-ZGNR with single vacancy defect located at the edge but under varying length, the disclosure of the findings from Figure 4 (b), Figure 6 (b) and Figure 8 (b) reveal the sub-band structure is not at all affected by varying length. In fact, varying length only affects the Green's function DOS. Non-ideal 4-ZGNR with single vacancy defect under varying length shows metallic properties as band gap is. The peak value for these sub-band occur at $\pm 8 \mathrm{eV}$. Furthermore, Figure 5 (b), Figure 7 (b) and Figure 9 (b) of 4-ZGNR with single vacancy defect located in the bulk but under varying length, also show similar characteristics as previously mentioned. Key differences are noticeable within the DOS for 4-ZGNR with single vacancy defect located at the edge and in the bulk. The highest peaks inside DOS of Figure 4 (c) to Figure 9(c) rise with length. Likewise, the number of peaks also increased.

\section{CONCLUSION}

Influence of single vacancy defect at varying length on electronic properties ... (Kien Liong Wong) 
In this paper, the electronic properties of a tight binding model of GNR with single vacancy is studied. The tight binding models is generated for 4-ZGNRs under varying length containing a single vacancy defect at different atomic structure locations. The Hamiltonian matrix was modified by identifying the location where defects occurred and eliminating tight binding energies. Non-ideal 4-ZGNRs' electronic properties such as Green's function DOS and sub-band structure are computed. Varying the length and position of the single vacancy defect on 4-ZGNRs' atomic structure has no effect on the sub-band structure but does have an profound implication on DOS as the Hamiltonian operator is used for Green's function computation.

\section{ACKNOWLEDGMENTS}

Kien Liong would like to express his appreciation for the award of Zamalah PhD Scholarship from the School of Graduate Studies Universiti Teknologi Malaysia (UTM). The authors acknowledge financial support from Fundamental Research Grant Scheme (FRGS) (Vote no.: R.J130000.7851.5F043) from the Ministry of Higher Education that allowed the research to proceed smoothly. Michael Tan acknowledges the support of the UTM Research Management Centre (RMC) for providing an excellent environment which allowed his result-oriented team to deliver results of high standards worthy of consideration by the industry.

\section{REFERENCES}

[1] Fujita M., Wakabayashi K, Nakada K., and Kusakabe K., "Peculiar Localized State at Zigzag Graphite Edge," Journal of the Physical Society of Japan, vol. 65(7), pp. 1920-3, 1996.

[2] Soldano C, Talapatra S., and Kar S., "Carbon nanotubes and graphene nanoribbons: Potentials for nanoscale electrical interconnects,” Electronics, vol. 2(3), pp. 280-314, 2013.

[3] Allen MJ, Tung VC, and Kaner RB, "Honeycomb Carbon: A Review of Graphene," Chemical Reviews, vol. 110(1), pp. 132-45, 2009.

[4] Zeng H, Leburton J-P, Xu Y., and Wei J., "Defect symmetry influence on electronic transport of zigzag nanoribbons," Nanoscale research letters, vol. 6(1), p. 254, 2011.

[5] Terrones H, Lv R, Terrones M, and Dresselhaus MS., "The role of defects and doping in 2D graphene sheets and 1D nanoribbons," Reports on Progress in Physics, vol. 75(6):062501, 2012.

[6] Datta S., "Nanoscale device modeling: the Green's function method," Superlattices and Microstructures, vol. 28(4), pp. 253-78, 2000.

[7] Zhang W, Lu W-C, Zhang H-X, Ho K., and Wang C., "Tight-binding calculation studies of vacancy and adatom defects in grapheme," Journal of Physics: Condensed Matter, vol. 28(11):115001, 2016.

[8] Wong KL, Chuan MW, Chong WK, Alias NE, Hamzah A, Lim CS, and Tan MLP., "Modeling of low-dimensional pristine and vacancy incorporated graphene nanoribbons using tight binding model and their electronic structures," Advances in Nano Research, vol. 7(3), pp. 207-19, 2019.

[9] Datta S., Lessons from Nanoelectronics: A New Perspective on Transport-Part A: Basic Concepts. World Scientific, 2017.

[10] Datta S., Electronic transport in mesoscopic systems. Cambridge university press, 1997.

[11] Wang C-Z, Lu W-C, Yao Y-X, Li J, Yip S., and Ho K-M., "Tight-binding Hamiltonian from first-principles calculations," Scientific Modeling and Simulation SMNS, vol. 15(1-3), pp. 81-95, 2008.

[12] Wong KL, Mahadzir MAS, Chong WK, Rusli MS, Lim CS, and Tan MLP, "Graphene Nanoribbon Simulator of Vacancy Defects On Electronic Structure," Indonesian Journal of Electrical Engineering and Informatics (IJEEI), vol. 6(3), pp. 265-73, 2018.

[13] Goh E, Chin HC, Wong KL, Indra ISB, and Tan MLP, "Modeling and Simulation of the Electronic Properties in Graphene Nanoribbons of Varying Widths and Lengths Using Tight-Binding Hamiltonian," Journal of Nanoelectronics and Optoelectronics, vol. 13(2), pp. 289-300, 2018.

[14] Indra ISB, Chin HC, Wong KL, Goh E, Lim CS, and Tan MLP, "Graphene Nanoribbon Simulator of Electronic Properties Using MATLAB," Journal of Nanoelectronics and Optoelectronics, vol. 13(3), pp. 405-14, 2018.

[15] Reich S, Maultzsch J, Thomsen C., and Ordejón P., "Tight-binding description of grapheme," Phys Rev B., vol. 66(3):035412, 2002.

[16] Wakabayashi K, Takane Y, Yamamoto M., and Sigrist M., "Electronic transport properties of graphene nanoribbons," New Journal of Physics, vol. 11(9):095016, 2009.

[17] Chin S-K, Lam K-T, Seah D., and Liang G., "Quantum transport simulations of graphene nanoribbon devices using Dirac equation calibrated with tight-binding $\pi$-bond model," Nanoscale Research Letters, vol. 7(1), p. 114, 2012.

[18] Datta S., "The non-equilibrium Green's function (NEGF) formalism: An elementary introduction," Electron Devices Meeting, 2002 IEDM '02 Digest International, 2002.

[19] Cresti A, Nemec N, Biel B, Niebler G, Triozon F, Cuniberti G., and Roche SJNR., "Charge transport in disordered graphene-based low dimensional materials," Nano Research, vol. 1(5), pp. 361-94, 2008.

[20] Maffucci A. and Miano G., "Electrical Properties of Graphene for Interconnect Applications," Applied Sciences, vol. 4(2), pp. 305-17, 2014.

[21] Wimmer M, Adagideli İ, Berber S, Tománek D., and Richter K., "Spin Currents in Rough Graphene Nanoribbons: Universal Fluctuations and Spin Injection," Physical Review Letters, vol. 100(17):177207, 2008.

Indo. J. Elec. Eng. \& Inf, Vol.7, No. 2, June 2019: 366 - 374 


\section{BIOGRAPHY OF AUTHORS}

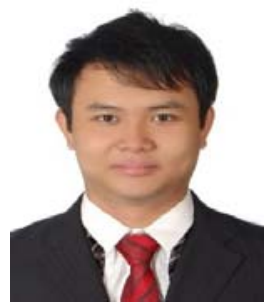

Kien Liong Wong was born in Skudai, Johor, Malaysia in 1993. He received the B. Eng. (electrical-electronics) degree from Universiti Teknologi Malaysia (UTM), Skudai, Malaysia, in 2017 and is currently pursuing the Ph.D degree in electrical engineering at Universiti Teknologi Malaysia (UTM), Skudai, Malaysia. He is conducting the research on the tight-binding quantum transport in graphene nanoribbons (GNRs) with line edge roughness and doping. His research interests are in graphene nanoribbons (GNRs), field effect trsnsistor (FET) devices. Previously, he did his internship at Venture GES Manufacturing Services (M) SDN BHD at Senai, Johor, Malaysia. He had published Graphene Nanoribbon Simulator of Vacancy Defects on Electronic Structure (Indonesian Journal of Electrical Engineering and Informatics (IJEEI), 2018), Modelling of Low-dimensional Lattice Hamiltonian for Electronic Properties of Single Vacancy Defect Graphene Nanoribbons (International Graduate Conference on Engineering, Science and Humanities (IGCESH), 2018) and others.

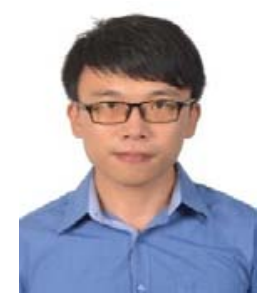

Mu Wen Chuan was born in Melaka, Malaysia in 1994. He received B.Eng. (electricalelectronics) degree from Universiti Teknologi Malaysia, in 2018. He is currently pursuing the $\mathrm{Ph} . \mathrm{D}$ degree in electrical engineering at Universiti Teknologi Malaysia. His research interests are in device modelling and circuit simulation of MOSFET devices.

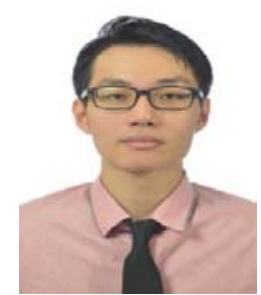

Chong Wee Khang was born in Muar, Johor, Malaysia in 1995. He received his B.Eng (electrical - electronics) degree with First - Class Honours from Universiti Teknology Malaysia (UTM), Skudai, Malaysia in 2018. His final year project was about the research on modelling of low dimensional lattice Hamiltonian for vacancy defects in graphene nanoribbons. He is currently pursuing his career in Oppstar Technology Sdn Bhd, Penang, as IC Design Engineer.

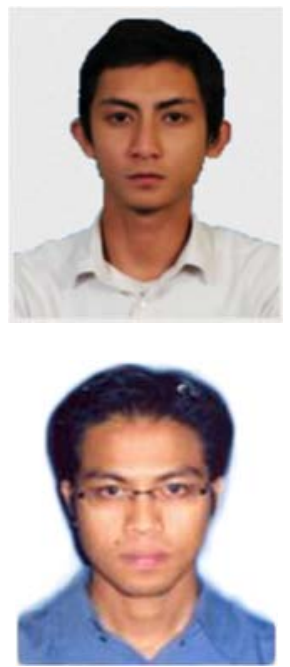

Afiq Hamzah received his B. Eng (2011), M. Eng (2014) and PhD (2018) in Electrical and Electronics Engineering from the Universiti Teknologi Malaysia. He is currently a senior lecturer with the School of Electrical Engineering, Universiti Teknologi Malaysia, Malaysia. His research interest includes virtual fabrication process, device compact modeling of multiple-gate FETs and graphene-based devices, and circuit simulation.

Mohd Shahrizal Rusli received his Ph.D in Electrical Engineering (2016), M. Eng. (Electronic and Telecommunication) (2010) and B. Eng. in Computer (2006) degree from Universiti Teknologi Malaysia (UTM), Johor. Between 2014 and 2015, he was attached to University of California Irvine, USA and University of Catania, Italy under the supervision of Prof. Dr. Nader Bagherzadeh and Assoc. Prof. Dr. Maurizio Palesi, respectively. He currently serves as senior lecturer at the Division of Electronic and Computer Engineering, UTM where his research interest and specialization are in the field of embedded processor system, deep learning application in embedded system, low-power and energy management in network-on-chip and wireless network-on-chip. He has published several journals and proceedings. He has been appointed as a reviewer for several journals. He is also a researcher in VLSI and Embedded Computing Architecture Design (VeCAD) Research Group and has participated in several courses and seminars. 

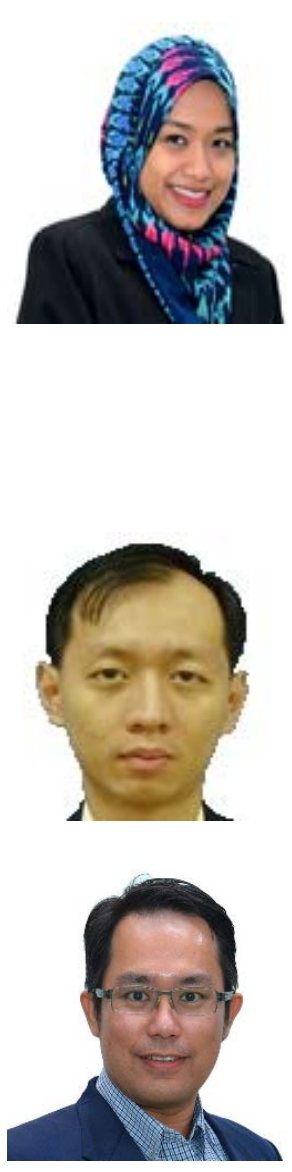

Nurul Ezaila Alias received her B. Eng. (Hons) degree in electrical and electronics engineering from Universiti Teknologi MARA, Malaysia (2007), M. Eng degree in Electronics \& Telecommunication Engineering from Universiti Teknologi Malaysia, Malaysia (2010). She has then received her $\mathrm{PhD}$ degree in Electrical Engineering \& Information System from The University of Tokyo (2014). She is currently a Senior Lecturer with the Dept. of Electronic and Computer Engineering, Universiti Teknologi Malaysia, Malaysia. She is a member of IEEE Society Malaysia Section, IEEE Electron Devices Society and The Institution of Engineers, Malaysia (IEM). She is in the reviewer of several International Journals, reviewer for IEEE Transactions on Very Large-Scale Integration (VLSI) Systems for 2 years until now. She is also very active in supervising postgraduate students both local and international students. Her research interests include advanced TCAD simulations and analysis of semiconductor devices, design techniques of FET-based Memory Application, Variability and Reliability Issue in Nanoscale FET Device. Reliability issue involves simulation and measurement framework of NBTI degradation. Lifetime prediction for improving the reliability challenges in the industry.

Cheng Siong Lim received his B. Eng. degree in Electrical Engineering (first class honors), M. Eng. degree (Electrical), and Ph.D. degree from Universiti Teknologi Malaysia (UTM), in 1999, 2004, and 2011, respectively. He is a senior lecturer in the School of Electrical Engineering, Faculty of Engineering, UTM. His research interests are in embedded system, emergency medical services, telerobotics and multi-agent system.

Michael Loong Peng Tan was born in Bukit Mertajam, Penang, Malaysia, in 1981. He obtained his Bachelor of Engineering and was appointed as a tutor in the same university in September 2003. He started his Master by research on June 2004 and carried out his research as a graduate intern in Penang Design Centre (PDC) Intel Technology Sdn. Bhd, Penang. He was awarded Masters of Engineering from UTM in 2006. Subsequently, Michael pursued his $\mathrm{PhD}$ in Engineering at Queens' College, Cambridge from October 2007 until June 2011. He was awarded his PhD degree in July 2011 at the University of Cambridge, United Kingdom. Presently, he is a Senior Lecturer and researcher in the field of low dimensional nanoelectronics devices in the Computational Nanoelectronics (CoNE) Research Group, School of of Electrical Engineering, Universiti Teknologi Malaysia. In addition, he is a Chartered Engineer registered under the Engineering Council, United Kingdom. He is a lifetime corporate member of the Institution of Engineers Malaysia (IEM), member of the Institution of Engineering and Technology (IET) and Institute of Physics (IOP), life member of the American Physical Society (APS) and senior member of IEEE (SMIEEE). His research interests are in device modeling and circuit simulation of silicon and carbon-based nanotransistor. 